\title{
Corela
}

Cognition, représentation, langage

HS-31 | 2020

Métalinguistiques.

\section{De l'(in)constance du métalinguistique dans un corpus de vulgarisation médicale}

\section{Valérie Delavigne}

\section{(2) OpenEdition}

\section{Journals}

Édition électronique

URL : http://journals.openedition.org/corela/11031

DOI : 10.4000/corela. 11031

ISSN : 1638-573X

Éditeur

Cercle linguistique du Centre et de l'Ouest - CerLICO

Référence électronique

Valérie Delavigne, «De l'(in)constance du métalinguistique dans un corpus de vulgarisation médicale », Corela [En ligne], HS-31 | 2020, mis en ligne le 04 juin 2020, consulté le 03 juillet 2020. URL : http://journals.openedition.org/corela/11031 ; DOI : https://doi.org/10.4000/corela.11031

Ce document a été généré automatiquement le 3 juillet 2020

\section{(c) (i) (2)(2)}

Corela - cognition, représentation, langage est mis à disposition selon les termes de la licence Creative Commons Attribution - Pas d'Utilisation Commerciale - Partage dans les Mêmes Conditions 4.0 International. 


\title{
De l'(in)constance du métalinguistique dans un corpus de vulgarisation médicale
}

\author{
Valérie Delavigne
}

1 En tant qu'objet d'étude à relier à une pratique sociale, les terminologies peuvent être appréhendées de plusieurs manières. L'une d'entre elles est d'observer les termes lorsqu'ils s'échappent de leur domaine d'usage habituel. Formes d'une culture, les terminologies ont en effet affaire avec l'intériorité et l'extériorité des communautés discursives, opposition qui permet de penser des questions de voisinage, de proximité, de contact. On a beau faire, les termes jouent l'indiscipline et ne restent guère cantonnés à l'intérieur des sphères d'activité qui les voient naître. Lorsque l'on est entre soi, que l'on appartient à la même communauté, l'usage des mêmes mots auxquels on attribue un sens commun permet de se comprendre. Cependant, dès lors que le terme technique ou scientifique sort de son cercle habituel, la connivence s'efface. Il est intéressant de porter son regard là où les terminologies se dissolvent, se désagrègent : à leur surface de séparation. Car c'est aux frontières que les difficultés émergent ; les choses se passent au jointif, dans l'entre-deux : sans voisin, il n'y a pas grand-chose à négocier.

2 Afin de rétablir une intercompréhension, les discours destinés aux " non-experts " s'attachent à redonner du sens aux mots. Le travail de négociation discursive passe par des jeux de reformulation bien décrits par les analystes des discours de vulgarisation (Jacobi 1986, 1999; Mortureux 1982, 1988; Reboul-Touré 2004 notamment). Ces dénivellations sémiotiques s'emploient à faciliter l'émergence du sens des termes et laissent apparaitre diverses relations sémantiques (Rey-Debove 1997).

3 A partir d'une analyse contrastive d'un corpus de documents médicaux, brochures pour les patients atteints de cancer et forums de discussion, nous nous proposons d'explorer les mouvements des unités terminologiques. Il ne s'agit pas ici de rendre compte des usages différenciés des terminologies médicales, mais de centrer notre attention sur les marqueurs métadiscursifs qui les accompagnent. L'analyse contrastive de ce corpus 
montre une économie spécifique de chacun de ces discours, repérable par des choix lexicaux, énonciatifs et argumentatifs divers, mais aussi, et ce sera le cœur de notre propos, par des formes métalinguistiques tout à la fois semblables et spécifiques. Si le corpus révèle des usages certes attendus du métalinguistique comme outil explicatif, il semble bien qu'il ne s'y réduise pas.

\section{Une approche socioterminologique}

4 Cette étude s'ancre en socioterminologie, approche sociolinguistique de la terminologie, objet longtemps peu pris en compte par les linguistes (Gaudin 1993, 2003, 2005). Elle est proche des travaux linguistiques tournés vers l'analyse des discours spécialisés qui, sans revendiquer l'étiquette "socioterminologique ", s'en rapprochent néanmoins par bien des aspects (Mortureux 1988 et les travaux du Cediscor comme Moirand 1994, Moirand \& Beacco (dir.) 1995, Cusin-Berche (dir.) 2000, Reboul-Touré 1995, 2004, ou encore Dury \& Picton 2009 ; voir aussi à ce propos Petit 2010). Ce travail s'inscrit dans une perspective d'analyse de discours qui tente de prendre en charge les enjeux de discours et en linguistique de corpus.

5 La terminologie a pour objet l'étude des termes, des systèmes de termes et des discours dans lesquels ils s'insèrent. Elle s'est développée indépendamment de la linguistique et ce n'est que récemment que les acquis de la linguistique et de la sociolinguistique ont été intégrés à la discipline. Devant des postulats historiquement légitimes mais devenus difficilement recevables, la socioterminologie a proposé des aménagements plus opératoires : les notions ne préexistent pas aux termes dans des systèmes notionnels ; les termes ne sont pas des objets en soi qui fonctionnent hors de toute énonciation; les domaines ne sont pas des lieux stables et clos qui ferrent les termes dans des sens immuables; les discours, et donc les corpus, sont au cœur des processus méthodologiques. C'est replacer ainsi au cœur de l'analyse un certain nombre d'acquis linguistiques.

6 Loin d'être une théorie constituée, la socioterminologie ne peut être qualifiée de discipline, tout au plus d'école de pensée. C'est en tout cas une autre manière d'envisager les terminologies qui naissent et vivent dans les organisations et les institutions, et possèdent chacune leurs spécificités. La socioterminologie s'attache à observer les unités terminologiques dans leur milieu, à examiner leur circulation, en prêtant une attention particulière aux questions d'usages, de validation sociale des termes, de normaison (Guespin 1993), de (dé)régulation. L'objectif est de décrire la variation des terminologies et des dispositifs énonciatifs qui les actualisent en prenant en compte les contraintes des structures organisationnelles ou institutionnelles où elles circulent et les conditions matérielles de la communication qui pèsent sur les formes.

\subsection{L'éloge des frontières}

7 Une des spécificités de la socioterminologie tient en son intérêt affirmé pour la circulation des termes (Gaudin 2003 ; Delavigne \& de Vecchi 2016). Cette perspective sociolinguistique mène à considérer les divers canaux par lesquels ils passent et s'oppose à la terminologie "classique " qui s'est longtemps focalisée sur les unités terminologiques circonscrites dans des domaines. Or bien des termes ne restent pas cantonnés aux communautés qui les ont vus naître. 
Ce constat de la circulation des termes n'est certes pas nouveau. Louis Guilbert y faisait déjà référence en son temps (Guilbert 1973 ; voir aussi Beacco 2000). Cependant, cette circulation s'accentue tout particulièrement aujourd'hui avec le développement d'internet. Notre monde s'est ouvert et les terminologies se retrouvent bien plus rapidement à l'extérieur des communautés discursives qui les actualisent d'ordinaire.

Que se passe-t-il lorsque les termes sortent de leur écosystème naturel ? La terminologie s'est peu intéressée aux problématiques de la vulgarisation, ce qui n'est pas le cas de la linguistique (Jacobi 1986; Mortureux 1982; Moirand \& Beacco 1995; Beacco 1995, 2000). Or c'est bien souvent aux bornes que naissent les difficultés. Faisons donc, pour reprendre le titre d'un opuscule de Régis Debray (2010), l'éloge des frontières. Cette posture, en permettant de penser des questions de voisinage, de proximité, de contacts, présente l'avantage, d'un point de vue méthodologique, de repérer les négociations terminologiques à l'œuvre en considérant les variations écrites et orales - diatopiques, diastratiques, diachroniques, etc. - en fonction des conditions de production, de circulation, de réception et d'interprétation. Ces discours à diffusion sociale large, parfois placés sous l'hyperonyme " discours de transmission des connaissances " (Moirand \& Beacco 1995), ont été fréquentés par les linguistes dont l'approche n'est pas spécifiquement terminologique, mais s'ancre plutôt en analyse du discours et en sémantique lexicale et discursive. Porter le regard vers ces discours amène à reconsidérer certains préceptes terminologiques et à replacer au cœur de l'analyse les pratiques sociales situées et les structures organisationnelles et institutionnelles qui contraignent l'énonciation.

\subsection{Corpus : caractérisation et méthode d'approche}

Notre analyse prend corps dans ce cadre. Ayant été amenée à intervenir dans le circuit de production de documents pour les patients atteints de cancer (Fervers et al. 2003 ; Carretier et al. 2010 ; Delavigne 2015), nous avons rassemblé des outils d'information pour les « patients $»^{1}$ : brochures papier et leurs « avant-textes » (Fenoglio \& Chanquoy 2007) dans lesquels sont colligés les tours d'écriture et les commentaires des scripteurs. Ce corpus sera désormais désigné par " corpus Brochures ». Viennent en contrepoint de ce corpus des discussions extraites de forums médicaux autour de la thématique du cancer, que nous désignerons par "corpus Forum» (pour une présentation plus approfondie du corpus, voir Delavigne 2013 et Delavigne 2017).

11 Ce qui justifie de rassembler ces deux genres de discours a priori hétérogènes est leur objectif similaire : l'information autour du cancer. La sphère numérique a transformé l'environnement social, économique et culturel contemporain. Le domaine médical n'est pas en reste : dans le panel des outils d'information sollicités par les patients, les outils numériques occupent désormais une place centrale. Les thématiques de santé ont ainsi envahi l'espace public, au point qu'Hélène Romeyer parle d'«industries de contenu» (2008). Contournant le modèle traditionnel d'information descendant du médecin vers le patient, le patient recherche aujourd'hui activement des informations. Il s'informe autrement, par le biais d'autres communautés et les formes d'éditorialisation de l'information s'en voient modifiées (Battaïa 2012). Les forums cristallisent cette individualisation des pratiques informationnelles médicales. Ces évolutions amènent à réviser les rapports entre les différents types de savoirs et ne sont pas sans répercussions sur les usages langagiers. 
12 Notre intérêt pour cet observable émane de l'hypothèse que le forum pouvait constituer un outil pour documenter un dictionnaire pour les patients. Nous avons montré ailleurs comment s'y aménage la diffusion de terminologies médicales. L'autodidaxie trouve là son chemin: on y voit une "culture périmédicale " se construire et s'imprimer dans la matérialité discursive (Delavigne 2013 ; à paraître), même si l'on sait que la recherche d'informations y est subordonnée à d'autres objectifs : quête de soutien, échanges et conseils pratiques (Romeyer 2008).

13 Les spécificités de cet objet discursif, qui correspond à une pratique sémiotique particulière, interrogent un certain nombre de concepts linguistiques de niveaux différents, comme les notions de genre discursif (le forum est-il un genre ?) ou de vulgarisation (peut-on catégoriser le forum comme un discours de vulgarisation?) (Colin \& Mourlhon-Dallies 2004; Panckhurst 2007; Anis 2009; Liénard \& Zlitni 2015), voire questionnent son statut même de texte (Paveau 2015). Même si notre approche reste «logocentrée », pour reprendre le terme de Marie-Anne Paveau, puisque nous ne prenons pas en compte les dimensions techniques et visuelles du forum, a-t-on les outils théoriques et méthodologiques pour se confronter à ce type de dispositif discursif? En effet, si l'on se penche sur la caractérisation formelle du forum médical, on se retrouve face à des formes spécifiques liées aux conditions matérielles de production de ces discours. Le forum est truffé de variables d'expression de «bas niveau " : ponctuèmes, emphase, focalisation des capitales ou graisse typographique, smileys et autres marqueurs d'émotions et de relations sociales (Marcoccia 2004). Les formes linguistiques qu'il convoque, ses écarts à la norme, son hétérogénéité sémiotique, son hybridation entre écrit et oral, ses particularités morphologiques, syntaxiques et énonciatives: néographies, abréviatifs, accidents dactylographiques, ellipses, poids de certains déictiques, tout comme ses caractéristiques discursives: délinéarisation, fragmentation, etc. (Anis 1998; Colin \& Mourlhon-Dallies 1995; Mourlhon-Dallies 2010), légitiment que l'on passe par une autre notion que Laroussi et Liénard désignent par «écrilecte» (2013). Ces aspects formels ne sont pas sans poser problème dès lors que l'on souhaite soumettre des fils de discussion à un outil d'analyse automatique.

$14 \mathrm{Au}$ delà de cette composante numérique, une des difficultés consiste à caractériser l'ensemble de ce corpus contrasté en gardant à l'esprit son hétérogénéité, caractéristique guidée par notre visée comparative. Nous avons convoqué dans notre titre le syntagme « discours de vulgarisation ». Il faut reconnaître que si la désignation est commode, elle fait fi de la spécificité des discours du corpus et gomme des différences de perspective. Ce corpus présente certes des traits communs aux discours de vulgarisation, notamment par les traces de didacticité sur lesquelles nous reviendrons largement plus bas; c'est ce qui nous pousse à conserver la désignation. Cependant, nous sommes ici dans une vulgarisation spécifique qui nécessite de penser au delà de genres discursifs clairement identifiables, entendus comme normes de pratiques (l'article de Science \& Vie ou celui de Sciences et Avenir par exemple, qui semblent être les prototypes du texte de vulgarisation), voire des répartitions à l'instar de celles que propose Petit (2010) par exemple, distribuées entre discours scientifiques (académiques), discours professionnels qui rassemblent les interactions entre spécialistes, et discours d'un "troisième type " qui sortent du champ de référence ${ }^{2}$. Nous pourrions catégoriser le corpus sous la désignation « discours de transmission des connaissances » (Moirand \& Beacco 1995); nos observations rejoignent en effet bien des 
pans des études menées sur ce type de discours comme le travail de négociation discursive qui accompagne le mouvement des termes. Cependant, cette catégorisation large gomme là aussi les spécificités des textes présents dans le corpus. Ce qui est sûr, c'est que le corpus questionne bon nombre de catégories linguistiques.

Conformément à certaines approches de l'analyse de discours, notre méthode pour aborder le corpus reste classique: notre problématique terminologique nous pousse naturellement à constituer le terme comme point d'entrée en tant que « pivot ». Notre définition du terme est socioterminologique : c'est une unité lexicale dont la spécificité est à relier à son statut dans une communauté discursive donnée. Par exemple, un « lit » dans le monde hospitalier n'est pas nécessairement un meuble. Le statut terminologique dépend donc du locuteur ou de l'analyste. Le terme est au sens de Rastier (2011) un passage qui renvoie à une intertextualité et à des cultures. Entrer dans le corpus par le terme permet de se focaliser sur certains évènements discursifs qu'il cristallise comme les commentaires méta- et épilinguistiques.

Nous avons utilisé un outil d'exploration de corpus, NooJ, développé par Max Silberztein ${ }^{3}$. Nous n'exposerons pas les différentes approches outillées disponibles pour analyser le matériau langagier. Disons simplement que le choix de NooJ se fonde sur le fait de pouvoir créer nos propres patrons de recherche sans l'effet de «boîte noire » de certains logiciels. Si les résultats obtenus ne sauraient avoir valeur de preuve et restent réductibles au corpus, l'utilisation d'un logiciel fait émerger des faisceaux de faits et met au jour des modes de fonctionnement spécifiques. Une exploration contrastive des corpus permet en outre de repérer des phénomènes qui n'auraient pas été décelés manuellement et de caractériser des régularités. Il faut cependant souligner la difficulté d'utiliser cet outil pour le corpus Forums dans la mesure où les caractéristiques évoquées plus haut en limitent l'analyse. Néanmoins, se confronter aux corpus de façon systématisée permet de mettre au jour les marqueurs mobilisés, d'en répertorier les usages et d'en dessiner le jeu.

\section{Marque, trace, marqueur et autres désignations : hétérogénéité terminologique}

17 Afin de donner accès au sens des unités terminologiques, les discours de vulgarisation convoquent un certain nombre de «structures doubles» (Fuchs 1982), énoncés paraphrastiques de «discours-sources» qui sont autant de «"proposition équationnelle" (...) : $\mathrm{Z}=\mathrm{X}$, où $\mathrm{Z}$ est un terme scientifique, et $\mathrm{X}$ une paraphrase non marquée scientifiquement" (Mortureux 1982). C'en est une des principales caractéristiques (même si la vulgarisation la meilleure est sans doute celle qui laisse oublier ces reformulations). Ces structures sont repérables par des segments linguistiques spécifiques:

Les marqueurs comme "c'est-à-dire", à savoir", "cela signifie que", "ou" (conjonction), indiquent le point de l'énoncé où la reformulation se réalise. Le marqueur fonctionne comme une césure ; il coupe l'énoncé qui est aussitôt repris et dit autrement sous équivalence sémantique. (Peytard 1993)

Ces marqueurs métalinguistiques ont été bien identifiés, au point qu'ils ont pu être constitués ailleurs en outils de repérage d'unités terminologiques sur d'autres corpus de discours de vulgarisation (Delavigne 2001). 


\subsection{Hétérogénéité désignationnelle}

$19 \mathrm{Au}$ seuil de ce parcours, nous voudrions interroger rapidement la notion de «marqueur ». Un marqueur signale une "activité » linguistique qui peut s'actualiser diversement. Prenons deux exemples pour illustrer notre propos : le suffixe-ette est un marqueur morphologique de petitesse; un accent est un marqueur sociolinguistique. Sur le plan descriptif, la conséquence de cette assiette sémantique accueillante est le flou terminologique par lequel la notion est désignée: marque, marqueur, marquage, trace, indice... qui se traduit par des structures, patrons, relateurs, motifs, constructions, expressions, routines ${ }^{4} . .$. autant de traces d'opérations, d'activités... La variation est prégnante et le paradigme désignationnel extrêmement étendu selon la fonction que l'on donne à ce "marqueur ». On peut voir dans cette inflation désignative la centralité de la notion en linguistique qui lui a consacré plusieurs colloques. Son sens se voit borné par diverses qualifications comme ici par métalinguistique, métadiscursif, métalangagier. Mais là encore, des alternances se repèrent, parfois chez les mêmes auteurs, selon les théories et le point de vue, selon que la forme actualise un commentaire ou qu'elle convoque une reformulation.

La notion de marqueur s'est développée dans d'autres communautés. On la repère par exemple dans les sciences de l'information et de la communication, qui la désignent préférentiellement par trace, avec une acuité particulière suscitée par les usages numériques (Galinon-Mélénec 2011; Jeanneret 2014; Pélissier 2015 entre autres). Analysant la notion, Yves Jeanneret insiste sur le travail réflexif nécessaire à son exploitation :

Seul ce travail de distinction catégorielle, en lieu et place d'une simple reprise plus ou moins allusive de la catégorie diffusante de la trace, permet d'envisager les nouveaux régimes de l'empreinte, de l'inscription et de l'écriture dans les médias informatisés (Jeanneret 2014: 3).

21 En lien direct avec une conception linguistique, le traitement automatique des langues et l'ingénierie des connaissances postulent que les marqueurs - désignés par patrons -, projetés sur des textes, vont permettre d'automatiser le repérage de terminologies et partant, de « connaissances » (Jacques \& Aussenac-Gilles 2006 par exemple), hypothèse que nous avons suivie par ailleurs (Delavigne 2001). Dans un monde langagier idéal, un triplet désignation/marqueur/désignation associerait de façon univoque un triplet désignation/relation/désignation. Cependant, devant la variation des marqueurs, ces disciplines mettent le doigt sur le fait qu'il faut laisser une place au genre, au contexte et à l'interprétation qu'on ne saurait réduire à une liste de métadonnées (AussenacGilles \& Condamines 2009).

Ce rapide détour permet de mettre le doigt sur la complexité et la polysémie de la notion. Pour notre part, nous conserverons le terme de marqueur dans la mesure où on trouve inscrit dans son sémantisme un évènement discursif, quel qu'il soit. Un marqueur est une marque, un témoignage, une trace d'une activité, d'une opération... dont il nous faudra affiner les fonctions.

\subsection{Hétérogénéité formelle et régularités}

Les marqueurs métalinguistiques peuvent être de diverse nature : marques typographiques (virgules, tirets ou parenthèses), verbes (être, appeler, nommer, signifier, 
désigner...), expressions spécifiquement métalinguistiques (c'est-à-dire, autrement dit, en d'autres termes...), joncteurs (ou, soit...), etc., tout cela étant cumulé parfois au sein du même énoncé :

La situation de chaque patient est donc discutée au cours d'une réunion appelée réunion de concertation pluridisciplinaire (RCP). ${ }^{5}$

En examinant les termes, institués comme unités pivots, NooJ nous a permis de localiser un certain nombre de ces structures. On remarque par exemple la forte densité sur le corpus des deux points, symptôme visible d'une importante activité reformulatrice. Cette densité est notamment la trace des glossaires présents dans chaque guide, les entrées étant suivies systématiquement de deux points; cependant ce marqueur graphique se retrouve également dans le corpus Forums, en alternance avec le signe $=$ :

RH - = recepteurs hormonaux negatifs

ki67: est 1 indice de prolifiration des cellules cancereuses

Her2: c est une proteine située ds la membrane des cellules; tu auras 1 injection d

herceptine pd qques mois. ${ }^{6}$

Remarquons de façon incidente que la fréquence des marqueurs de reformulation n'est pas identique entre les deux corpus. Guillemets de marquage autonymiques et parenthèses sont ainsi moins présents dans les forums. Être un par contre, forme de base de la prédication de l'identité, est un marqueur de reformulation omniprésent, même si, à l'instar d'autres corpus, il peut être équivoque ou, tout simplement, ne pas actualiser de prédication métalinguistique et introduire un simple commentaire sur l'objet de discours comme dans l'exemple suivant :

Ce petit coin du forum est un havre de paix pour nous

S'il y a certes multiplicité des formes, il y a dans le même temps constance de certaines unités convoquées. NooJ nous a permis de confirmer l'intuition que nous avions de la récurrence de plusieurs marqueurs métalinguistiques dans le corpus. Ces régularités nous ont amenée à intégrer la problématique des « routines » discursives telles que Née et al. les définissent :

Une routine discursive consiste en la mise en relation de séquences linguistiques récurrentes, partiellement figées (...) avec des déterminations discursives et des fonctions textuelles propres à un genre ou une sphère d'activité. (Née et al. 2014 :

2119)

27 Ainsi peut-on repérer des segments récurrents du type: " on parle de... ", " les médecins disent " ou "des patients disent», qui paraissent constituer une sorte de phraséologie du corpus Brochures. On retrouve là des «motifs » au sens de Longrée \& Mellet (2013), autrement dit des structures préformées qui combinent deux ou plusieurs unités. Certaines séquences vulgarisatrices sont plus fréquentes que d'autres, comme « parler [ADV] de » :

Lors de la découverte d'un cancer, il arrive ainsi que l'anatomopathologiste, le médecin en charge de l'analyse microscopique, n'arrive plus à définir l'origine de la cellule; on parle de tumeur « indifférenciée ».

On parle de forme sporadique lorsque ce sont ces mutations dites somatiques qui sont impliquées dans un cancer

On encore « $[\mathrm{ADV}]$ appelé » :

Les Kiosques d'accueil et d'information sur le cancer (parfois appelés KIC ou KAC) sont destinés aux personnes malades ou non, désireuses d'obtenir une information sur le cancer. 
Pour bénéficier de cette prise en charge à $100 \%$ dans le cadre de votre ALD, votre médecin traitant doit remplir un formulaire appelé Protocole de soins. faire négliger le lieu d'apparition des marqueurs métalinguistiques. En effet, nous faisions l'hypothèse qu'ils n'apparaissent pas n'importe où dans les textes. D'un point de vue méthodologique, cela implique de prendre en compte 1- la structure du texte, 2le lieu d'apparition du marqueur dans le tissu discursif. de marqueurs en tête de chapitre, ce que l'analyse a confirmé : les débuts de parties sont des lieux qui mobilisent une forte quantité de structures métalinguistiques dans la mesure où $\mathrm{y}$ apparaissent de nouvelles notions appelant une reformulation explicative. Mais les marqueurs essaiment également tout au long du texte. Ils n'apparaissent cependant pas n'importe où : dès qu'un nouveau terme que le scripteur estime opaque apparait, il se voit doté d'un appareillage métalinguistique destiné à en élucider le sens. Le texte ensuite "oublie» cette activité métalinguistique, le terme étant considéré comme acquis, inscrit dans la mémoire discursive du lecteur. Il entre alors de plain pied en usage dans le tissu textuel.

\section{Hétérogénéité fonctionnelles des marqueurs : variation de l'activité métalinguistique}

Les traces de reformulation se transforment en fonction des termes, de leurs lieux d'apparition, des genres textuels dans lesquels ils circulent, des supports où ils sont convoqués... Une des caractéristiques des marqueurs métalinguistiques est leur polysémie. D'un corpus à l'autre, ces éléments discursifs ont-ils les mêmes fonctions ?

\subsection{Un objectif prépondérant : la didacticité}

L'objectif le plus visible et le plus décrit des jeux de reformulation dans les discours de vulgarisation est de tenter d'établir une intercompréhension: un des rôles du métalinguistique est en effet de contribuer à des textes efficaces et adaptés au public considéré. C'est le projet même des guides pour les patients qui sont amenés à convoquer un certain nombre de termes spécialisés (INCa 2013). Faute de connivence, le sens de certaines unités doit être travaillé. A cette fin, des propositions équationnelles du type terme-marqueur-reformulant ou reformulant-marqueur-terme visent à faciliter l'émergence du sens de ces unités terminologiques, réputées obscures, en établissant une équivalence sémantique. Ces dénivellations sémiotiques actualisent des énoncés définitoires autour de ces unités, qui sont autant de traces de « didacticité » (Moirand 1992) ${ }^{7}$ : 
Un effet secondaire est une réponse indésirable à un médicament lorsqu'il est pris à des doses normales

Cette diminution [des composants du sang] entraîne souvent des effets secondaires : perte de cheveux, nausées, vomissements, mucites, diarrhées ou constipation, etc. précision du terme supposé non ou mal connu. Des gloses explicatives et définitoires, de formes variées - ici deux définitions, l'une en intension, l'autre en compréhension -, aménagent la sémantisation des unités terminologiques. Le rôle du marqueur métalinguistique est alors d'établir un pont sémantique à finalité didactique entre un terme et ses paradigmes définitionnels ou désignationnels (Mortureux 1982, 1988, 1993). Remarquons qu'il arrive que le marqueur s'efface, notamment en cas de diaphore, ce qui peut poser un problème méthodologique à l'analyste pour repérer les différents paradigmes.

Contrairement à ce qu'on pourrait attendre, le corpus Forums n'est pas exempt de ces traces de didacticité. Même si, dans une première approche, on peut imaginer que le discours scientifique et médical s'y dissout, il s'avère que c'est un lieu émaillé de terminologie médicale et de vulgarisation (Delavigne 2013). Les énoncés définitoires font florès :

chimio (...) : c'est une série de produits qu'ils te mettent en intra-veineuses

Marie, un clip axillaire est une petite agrafe métallique utilisée couramment par les

chirurgiens, pour stopper un saignement ou repérer un endroit préçis sur les radiographies

On y repère de nombreuses traces d'« activité définitoire spontanée » (Husson icimême), et celle-ci correspond souvent à une demande explicite :

Récepteurs d'oestrogènes Positifs $99 \%$ intensité $3+$

Récepteurs de progestérones Positifs $75 \%$ intensité 3+

HER-2 : Négatif : $1+$

J'aimerais qu'on m'explique tout ça un peu et qu'on me rassure, si possible...

? L'une d'entre vous peut -elle m'expliquer ce que veut dire :

" résultats ANAPATH"

Je ne trouve pas ce mot (anapath) dans le dico, mais peut être s'agit-il d'une abréviation

Ce que l'on voit ici émerger, ce sont donc d'autres formes de didacticité. Se met en place tout un jeu de questions-réponses directes ou indirectes, qui correspondent au régime « épistolaire » du forum (Colin \& Mourlhon-Dallies 2004).

Certes, on trouve dans le corpus Brochures des questions/réponses à visée didactique et proches des préoccupations de patients, qui vont au-delà de la «question rhétorique ». Citons en exemple les titres de paragraphes successifs autour de scanner:

Qu'est-ce qu'un scanner?

A quoi sert un scanner?

Qu'est-il nécessaire de faire avant un scanner?

Comment se déroule un scanner?

Le scanner est-il désagréable?

Quels sont les risques éventuels d'un scanner?

Quand obtient-on les résultats d'un scanner?

Dans les forums, le dispositif énonciatif permet des tours conversationnels directs bien qu'asynchrones. Outre de nombreuses formes narrativisées, on voit apparaître des citations de paroles de professionnels de santé du type "mon médecin m’a dit que... », d'autres paroles de patients, voire la mention de brochures: 
comme le dit le thiérachien suite a la rencontre avec l'oncologue il y a une réunion pluridisciplinaire de médecins

exemple liste

Réunions de Concertation Pluridisciplinaire avec l'oncologue

- Pneumologie $\cdot$ Gastro-entérologie $•$ Sénologie $•$ Gynécologie

- Urologie $\cdot$ Otorhinolaryngologie $\cdot$ Dermatologie

- Hématologie $\bullet$ Neuro-oncologie.

voir BROCHURE

reçus dans des consultations qui, structurellement, ne peuvent laisser que peu de temps à l'information ${ }^{8}$. Dès lors, ce sont les patients qui prennent le relais et transmettent les connaissances acquises sur le terrain de la maladie. Tout au long du corpus Forums apparaissent en effet ces formes didactiques spécifiques, convoquées par des patients pour des patients, mettant en œuvre une "vulgarisation fonctionnelle ». Elles posent la question de la qualité d'un discours de vulgarisation. Se profile par cette expertise terminologique une communauté de pratiques que nous avons appelée ailleurs «communauté d'aventure " à la suite de Louis Quéré (2003), définie en termes d'expérience partagée (Delavigne à paraitre). D'autres pratiques vulgarisatrices dans lesquelles se réaménagent les figures de l'interlocution se dessinent et laissent émerger d'autres enjeux.

\subsection{Cas particulier: quand le terme devient autonyme}

$\mathrm{Au}$ delà de cette intention didactique portée par les textes de nos deux corpus, les brochures pour les patients portent le projet programmatique d'un patient « acteur ", inscrit dans un paradigme participatif (Carretier et al. 2010). L'objectif revendiqué est d'élaborer des outils au service d'un dialogue qui doit s'établir entre les patients, leurs proches et les professionnels de santé. Le patient est censé «s'approprier » un certain nombre de termes médicaux pour ensuite être en mesure de les comprendre et, mieux encore, de les utiliser dans ses échanges avec les professionnels. Différentes stratégies, que ce soit au niveau macro ou microtextuel, sont mises œuvre par les scripteurs à cette fin d'équipement terminologique, profondément dialogique, stratégies au nombre 
desquelles on compte la mise en œuvre d'outils métalinguistiques que nous venons d'évoquer et, plus particulièrement, autonymiques. non-initié. vulgarisatrice : apparente.

mammotome.

Le marqueur métalinguistique peut ainsi avoir une fonction de monstration du terme. On le sait, les marqueurs métalinguistiques ne s'attachent pas à n'importe quel mot ; dans une perspective de vulgarisation, le couplage fonctionne a priori avec les termes scientifiques, techniques ou médicaux dont la forme en elle-même peut interpeller le

Le dispositif métalinguistique, signalant le non-isomorphisme d'un système terminologique et du lexique courant, organise un "arrêt sur mot ", pour reprendre l'expression de Jacqueline Authier-Revuz (2000), qui matérialise l'activité

Le terme neuropathique est composé de neuro «système nerveux» et pathique, qui

vient du grec pathos: «la souffrance». Ces douleurs surviennent sans raison

Neuropathique est une unité qu'Ad Hermans qualifie de terme "cryptique ", par opposition au terme "delphique (1997), autrement dit un terme non directement compréhensible: son sens n'est accessible que si le signe est connu. A l'inverse, les termes delphiques ont une apparence familière, mais leur interprétation se démarque de l'interprétation qui en est faite habituellement : ils nécessitent un «augure » pour accéder au bon sens. Autrement dit, ce sont des homonymes de lexies plus courantes, des termes construits par «néologie sémantique », par opposition aux termes formés par néologie formelle, dérivation ou composition. Parler du mot devient alors un outil d'appropriation terminologique dont témoigne l'organisation du tissu discursif :

On parle de laparotomie pour une ouverture de l'abdomen, de thoracotomie pour une ouverture du thorax.

La symétrie morphologique des termes laparotomie et thoracotomie mise ainsi en évidence contribue à en expliciter la forme. C'est là un autre type de didacticité, dans laquelle l'autonymie, qui vient souligner le signifiant, autorise dans le même temps par un effet définitoire l'appropriation du signifié.

Ces stratégies didactiques contribuent à créer un réseau intratextuel ou extratextuel. Un chaînage sémantique est mis en place, puis le terme est "lâché ", perdant son « escorte métalinguistique » pour reprendre le terme de Pascale Janot (ici-même). Dans les brochures, le lecteur n'est cependant jamais laissé complètement à lui-même : un astérisque accompagne le terme et lui signale qu'il peut se référer à un glossaire en fin d'ouvrage. Cet astérisque est un marqueur graphique métalinguistique à part entière, à l'instar des liens hypertextes sur internet qui conduisent à une définition ou une page de vulgarisation (Reboul-Touré 2004).

Ces termes se retrouvent ensuite sur les forums, accompagnés certes de commentaires métalinguistiques, mais aussi épilinguistiques, autrement dit relatifs au jugement linguistique du locuteur :

voilà les filles lundi j'ai passé ce curieux examen au drole de nom et vraiment je ne m'attendai pas à un tel moment

Mammotome, autre terme cryptique, voit sa forme exhibée, puis il est reformulé en « curieux examen » où l'hyperonyme examen fonctionne bien comme définisseur, mais dont la qualification par curieux insiste surtout sur l'expérience vécue. 


\subsection{Négocier les termes : mot juste, ajustement sémantique et polyphonie}

51 Dans la gamme des usages du métalinguistique, nous voudrions mettre l'accent sur un autre type de couplage discursif. Dans l'extrait suivant, le premier énoncé est une proposition d'un scripteur à un médecin, chargé de valider le contenu d'un guide pour les patients :

Ce guide présente la prise en charge des formes les plus fréquentes des cancers de la vessie : les tumeurs urothéliales. Les formes plus rares ne sont pas abordées. auquel l'expert, invité à commenter l'énoncé, répond ${ }^{9}$ :

Très grande hétérogénéité de tumeurs de vessie (carcinome urothélial). Certaines avec très peu de chance de récidive et de progression (LMP et PTA bas grade, elles sont communément appelées «polype «). Doit-on les appeler cancer ? et d'autres létales dans plus de $50 \%$ des cas (PT2 haut garde) (à proprement parlé K de la vessie).

53 L'association autonymique porte ici sur les mots de l'expert. Une négociation terminologique se met en place sur le choix des désignations : «Doit-on les appeler cancer?». "À proprement parlé K de la vessie » renvoie à la problématique du mot «juste ». Ce phénomène est exacerbé dans l'extrait suivant où le premier énoncé correspond là encore à une proposition du rédacteur :

Le néphroblastome est un cancer très différent du cancer du rein de l'adulte : les cellules impliquées ne sont pas les mêmes et les traitements sont donc différents. à laquelle le médecin réagit de la façon suivante :

Ce n'est pas le terme exact du cancer du rein de l'adulte. Le terme exact est carcinome à cellules rénales

On voit la culture médicale de l'expert prendre le pas sur l'objectif de vulgarisation. Cela va au delà d'une simple affaire de lexique. Ce qui s'exprime ici, c'est le sentiment qu'il n'existe qu'un seul "vrai terme" pour dire le réel. Cette problématique, récurrente chez les experts, peut se lire également comme une trace d'individuation linguistique. Ici, c'est l'institution médicale qui entre en scène et laisse poindre en filigrane une autre figure de l'interlocution, celle des pairs. L'expert, garant de la qualité des contenus médicaux des brochures, se sait soumis à leur jugement et se retrouve aux prises avec un double adressage.

On voit ainsi comment entrer dans le corpus par ces traces métalinguistiques permet de mettre le doigt sur la distance difficilement réductible entre discours experts et discours patients. Si certains termes subsistent dans les brochures, il faut y voir le symptôme de la difficulté pour le rédacteur de choisir son camp : supposé produire un document vulgarisé, il doit dans le même temps tenir compte de la diversité des voix et négocier les cultures. Pris dans un dialogisme intertextuel (les mots de l'autre, expert et garant de la fiabilité des informations) et interactionnel (les mots pour l'autre, le patient et ses proches), la difficulté est précisément de faire avec cette polyphonie.

57 Le corpus Forums n'est pas exempt de ces négociations autonymiques autour des termes :

Merci à toi de m'avoir éclairée un peu plus. En revanche dans un cancer métastasé je crois qu'on ne peut pas parler de guérison.

Guérison est ici ambigu : soit ce n'est pas le bon terme (on parle de rémission, non de guérison), soit il est inadéquat (il ne peut pas y avoir de guérison). Mais dans les deux 
cas de figure, l'enjeu est une affaire de validation terminologique, une négociation autour d'une normaison. Cette validation est modalisée cependant par croire qui manifeste les précautions prises par la patiente et convoque l'interdiscours. Ce terme guérison est bien sûr loin d'être anodin ; c'est même l'exemple type d'un mot chargé d'enjeux :

Le patient en a souvent conscience : il faut plusieurs années pour que les médecins parlent de guérison. En attendant, il s'agit d'une rémission. Et cette incertitude est difficile à gérer.

La notion de guérison fait l'objet d'un chapitre spécifique d'une brochure dont le titre actualise les deux isonymes: "Rémission, guérison». On retrouve autour des deux termes une abondance de formes autonymiques, traces du travail d'ajustement terminologique tenté par le scripteur :

Le mot guérison fait rarement partie du vocabulaire d'un médecin dans ses échanges avec ses patients traités pour un cancer. Il emploie plus volontiers le terme " rémission complète » parce qu'il sait qu'une récidive, même lointaine, est possible.

En revanche, le mot guérison se rencontre souvent dans les statistiques médicales. On lit par exemple qu'aujourd'hui, on guérit plus d'un cancer sur deux, alors qu'on n'en guérissait qu'un sur trois il y a trente ans. Comme toute moyenne, cette affirmation masque de grandes disparités selon les types de cancers. Mais l'emploi du mot guérison traduit ici en langage ordinaire une notion qu'on appelle «le taux de survie relative à 5 ans ».

Les statisticiens estiment en effet qu'un patient ayant eu un cancer a des chances élevées d'être «guéri »lorsque, 5 ans après le diagnostic, il retrouve la même espérance de vie que l'ensemble de la population de même âge, de même sexe et n'ayant pas eu de cancer.

Le corpus Forums se fait l'écho de ce type de développement :

Si tu lis les fiches des impatientes, tu en trouveras certaines qui ont eu des rémissions, même après des métastases. J'espère que ça sera ton cas. Mais tu sais, dans le cancer du sein spécifiquement, en l'état des connaissances actuelles, il y a pas de "guérison". On a juste des bonnes stats de survie à 10 ans...

61 Les guillemets de connotation autonymique signalent la négociation autour du terme guérison, suivi par la séquence " On a juste des bonnes stats de survie à 10 ans... », trace d'énoncé antérieur qui contribue à un effet définitoire. Nous pouvons repérer en effet des traces d'intertextualité prégnante, citations parfois au mot près de brochures pour les patients (Delavigne 2013).

62 Ce qui affleure de ces extraits, c'est une autre forme d'expertise, expertise qui peut s'accompagner de diverses précautions oratoires :

L'index de prolifération correspond au Ki, dont on parle parfois sur ce forum. C'est un peu le niveau d'agressivité de la tumeur.

Le marqueur métalinguistique «c'est» met en œuvre une recatégorisation terminologique, modalisée par « un peu » qui vient moduler la reformulation, montrer l'approximation du reformulant et peut-être la prudence du locuteur ${ }^{10}$. On peut rapprocher ce cotexte de celui que nous convoquions supra « je crois qu'on ne peut pas parler de guérison» dans lequel "je crois", trace de l'insécurité linguistique du locuteur, traduit son sentiment d'illégitimité. Se joue là sans doute une forme d'enjeu identitaire à l'œuvre dès lors que le terme technique apparaît, qui pose la question de la « garantie » du savoir convoqué (Rastier 2011). 
64 On voit ainsi émerger de nouvelles formes de connaissances, de nouvelles formes d'expertise. Cette expertise peut se décliner de deux manières. La première est celle des connaissances engrangées au fil des fréquentations des équipes médicales et des lectures (de brochures, d'ouvrages, de sites internet, de blogs, de forums...) ; la seconde est une expertise "expérientielle ", fondée sur l'expérience de la maladie. Si nous avons $\mathrm{pu}$ mettre en évidence cette expertise expérientielle par le biais du métalinguistique (cf. supra à propos de mammotome "ce curieux examen»), cette expertise est encore plus visible lorsque l'on se tourne vers les formes narrativisés de la maladie et de ses traitements, dimension interactionnelle qui reste à explorer.

\section{Pour conclure}

65 La catégorie du métalinguistique est un trait quasi-définitoire des discours de vulgarisation, sorte de "signature générique» (Legallois \& Tutin 2013: 10). En continuité avec ces analyses, nous avions dans un premier temps fait l'hypothèse de la constance de ces marqueurs sur notre corpus. Nous avions repéré en effet la régularité de certains segments, autant de solidarités lexicales et syntaxiques qui nous ont amenée à y voir des « routines » d'écriture (Delavigne 2017).

66 Si la didacticité est une fonction prévisible d'un corpus de brochures d'information pour les patients, elle est moins attendue sur un corpus de forums médicaux. Or, en examinant les deux corpus de manière contrapunctique, nous avons pu y reconduire le même type d'observations: la didacticité qui vise la dissolution du jargon est prépondérante; les marqueurs métalinguistiques mettent en scène des énoncés définitionnels, des reformulations, etc. Ce constat étoffe les lieux discursifs de la vulgarisation et, dans la continuité de ce que soulignait Sandrine Reboul (2004), vient élargir la gamme des formes qu'elle est susceptible de prendre.

67 Après avoir fait le constat de l'hétérogénéité formelle des marqueurs repérés sur le corpus, la variation fonctionnelle des formes discursives de reformulation est vite devenue une de nos questions de recherche. L'entrée dans le corpus par le métalinguistique attaché aux termes s'avère très productive. Le corpus déploie un panel varié de marqueurs métalinguistiques polyfonctionnels. Dans cet espace discursif spécifique, on ne peut pas réduire le métalinguistique à la seule visée illocutoire explicative ou reformulatrice et à son effet définitoire. Au-delà de la tentative de résolution de la fracture linguistique, l'analyse de l'activité métalinguistique permet de rendre compte de la façon dont les terminologies sont reprises, commentées, recatégorisées, validées, évaluées. Elle met en évidence une vulgarisation spécifique, adaptée et déclarée comme telle. Et elle permet de voir comment les forums constituent un espace qui reconfigure la figure de l'expert en aménageant un espace de validation où se construisent diverses identités, et notamment celle de "passeur " d'expérience. 


\section{BIBLIOGRAPHIE}

Anis J. (1998). Texte et ordinateur : l'écriture réinventée ? Paris : De Boeck Supérieur.

Anis J. (dir.) (2009). Communication et langue française. Paris : Hermès.

Appel Corpus 15 (2015). « Segments et séquences textuelles : méthodologie et caractérisation ».

Aussenac-Gilles N. \& Condamines A. (2009). Variation syntaxique et contextuelle dans la mise au point de patrons de relations sémantiques. In Minel J.-L. (dir.). Filtrage sémantique. Paris : Hermès/Lavoisier. 115-149

Authier J. (1982). La mise en scène de la communication dans des discours de vulgarisation scientifique. Langue Française 53. 34-47.

Battaïa C. (2012). L'analyse de l'émotion dans les forums de santé. Actes de la conférence conjointe JEP-TALN-RECITAL. RECITAL. 267-280.

Beacco J.-C. (1995). À propos de la structuration des communautés discursives : beaux-arts et appréciatif ». Les Carnets du Cediscor 3. 136-153.

Beacco J.-C. (2000). Écritures de la science dans les médias. Les Carnets du Cediscor 6. 15-24.

Carretier J., Delavigne V. \& Fervers B. (2010). Du langage expert au langage patient : vers une prise en compte des préférences des patients dans la démarche informationnelle entre les professionnels de santé et les patients. Sciences-Croisées 6. En ligne : http://sciencescroisees.com/N6/Carretier.pdf

Chaurand J. \& Mazière F. (dir.) (1990). La définition. Paris : Larousse.

Colin J.-Y. \& Mourlhon-Dallies F. (2004). Du courrier des lecteurs aux forums de discussion sur l'internet : retour sur la notion de genre. Les Carnets du Cediscor 8. 113-140.

Cusin-Berche F. (dir.) (2000). Rencontres discursives entre sciences et politiques dans les médias. Les Carnets du Cediscor 6. 9-11.

Debray R. (2010). Éloge des frontières. Paris : Gallimard.

Delavigne V. (2001). Repérage de termes dans un corpus de vulgarisation : aspects méthodologiques. Actes des quatrièmes rencontres « Terminologie et Intelligence artificielle». Nancy : 3-4 mai 2001. 33-43.

Delavigne V. (2013). Les forums médicaux, une ressource pour la lexicographie? Texte et Corpus 5 (Actes des Journées de Linguistique de Corpus 2013). En ligne : http://www.licornubs.com/jlc7/ACTES/Delavigne_JLC13.pdf

Delavigne V. (2015). Quand le linguiste devient acteur : vulgarisation médicale et prescription linguistique. In Caron F. et al. (dir.). Cultures de recherche en linguistique appliquée. Paris : Riveneuve Edition. 77-95

Delavigne V. (2017). L'écriture pour les patients : une écriture singulière ? Pratiques 173-174.

Delavigne V. (à paraître). Une analyse socioterminologique de forums de patients atteints de cancer : culture périmédicale et expertise . In Simon E., Arborio S., Halloy A. \& Hejoaka F. (dir.). Les savoirs d'expérience en santé. Défis épistémologiques et enjeux identitaires. Nancy : Presses universitaires de Nancy (Questions de communication. Série actes). 
Delavigne V. \& Vecchi D. de (2016). Socioterminologie et pragmaterminologie : rencontres et complémentarités. In Roche C. (dir.). Terminologie \& Ontologie : théories et applications. Chambéry : Éditions de l'Université Savoie Mont Blanc (Terminologica).

Ducrot O. (2002). Quand peu et un peu semblent coorientés : peu après et un peu après. Cahiers de linguistique française 24. 207-229.

Dury P. \& Picton A. (2009). Terminologie et diachronie : vers une réconciliation théorique et méthodologique? Revue Française de Linguistique Appliquée XIV(2). 31-41

Fenoglio I. \& Chanquoy L. (2007). Avant-propos. La notion d'"avant-texte" : point de rencontre pour une compréhension de l'écriture en acte. Langue française 155. 3-7.

Fervers B., Leichtnam-Dugarin L., Carretier J., Delavigne V., Hoarau H., Brusco S. \& Philip T. (2003). The SOR SAVOIR PATIENT project-an evidence-based patient information and education project. British Journal of Cancer 89(S1). S111-S116.

Fuchs C. (1982). La paraphrase entre la langue et le discours. Langue Française 53. 22-33.

Fuchs C. (1994). Paraphrase et énonciation. Gap : Ophrys.

Galinon-Mélénec B. (dir.) (2011). L'homme trace : perspectives anthropologiques des traces contemporaines. Paris : CNRS éditions.

Gaudin F. (1993). Pour une socioterminologie : des problèmes sémantiques aux pratiques institutionnelles. Publications de l'Université de Rouen.

Gaudin F. (2003). Socioterminologie : une approche sociolinguistique de la terminologie. Bruxelles : De Boeck-Duculot.

Gaudin F. (2005). La socioterminologie ». Langages 157. 81-93.

Guespin L. (1993). Normaliser ou standardiser. Le Langage et l'homme XXVIII 4. 213-222.

Guilbert L. (1973). La spécificité du terme scientifique et technique ». Les vocabulaires techniques et scientifiques. Langue française 17.5-17.

INCA, Institut National du Cancer (2013). Cancer info : méthodologie d'élaboration des contenus. En ligne : http://www.e-cancer.fr/Patients-et-proches/Cancer-Info

Longrée D. \& Mellet s. (2013) Le motif : une unité phraséologique englobante ? Étendre le champ de la phraséologie de la langue au discours. Langages 189. 65-79.

Hermans A. (1997). Mots et termes en sociologie. In Boisson C. \& Thoiron P. (dir.). Autour de la dénomination. Lyon : Presses universitaires de Lyon. 269-278.

Jacques M.-P. \& Aussenac-Gilles N. (2006). Variabilité des performances des outils de TAL et genre textuel ». TAL 47. En ligne : https://www.atala.org/Variabilite-des-performances-des

Jacobi D. (1986). Diffusion et vulgarisation. Itinéraires du texte scientifique. Paris : Les Belles Lettres.

Jacobi D. (1999). La communication scientifique. Discours, figures, modèles. Grenoble : Presses Universitaires de Grenoble.

Jeanneret Y. (2014). La fabrique de la trace : une entreprise herméneutique. In Idjeraoui-Ravez L., Pélissier N. \& Galinon-Mélénec B. (dir.). Quand les traces communiquent... Culture, patrimoine, médiatisation de la mémoire. Paris : L'Harmattan. 47-64.

Laroussi F. \& Liénard F. (2013). Des écrans à la rue : l'émergence de communautés sociolinguistiques virtuelles pendant la révolution tunisienne ». In Daghmi F., Toumi F. \& 
Amsidder A. (dir.). Les médias font-ils les révolutions ? Regards critiques sur les soulèvements arabes. Paris : Harmattan. 289-297.

Legallois D. \& Tutin A. (2013). Présentation : vers une extension du domaine de la phraséologie. Langages 189. 3-25.

Liénard F. \& Zlitni S. (dir.) (2015). La communication électronique. Enjeux, stratégies, opportunités. Limoges : Lambert Lucas.

Marcoccia M. (2004). La communication écrite médiatisée par ordinateur : faire du face à face avec de l'écrit. Journée d'étude de l'ATALA « Le traitement automatique des nouvelles formes de communication écrite (e-mails, forums, chats, SMS, etc.) ». En ligne : http://sites.univprovence.fr/ veronis/je-nfce/Marcoccia.pdf

Moirand S. (1992). Autour de la notion de didacticité. Les Carnets du Cediscor 1. 9-20.

Moirand S. \& Beacco J.-C. (1995). Autour des discours de transmission des connaissances. Langages 117. 32-53.

Mortureux M.-F (1982). Paraphrase et Métalangage dans le dialogue de vulgarisation. Langue Française 53. 48-61.

Mortureux M.-F. (1988). La vulgarisation scientifique : parole médiane ou dédoublée. In D. Jacobi \& B. Schiele (dir.). Vulgariser la science. Le procès de l'ignorance. Seyssel : Champ Vallon. 118-148.

Mortureux M.-F. (1993). Didacticité et discours “ordinaire”. Les Carnets du Cediscor 1. 21-31.

Mourlhon-Dallies F. (2010). Modifications et inventions graphiques dans les écritures électroniques. Le français aujourd'hui 170. 101-112.

Née E., Sitri F. \& Veniard M. (2014). Pour une approche des routines discursives dans les écrits professionnels. SHS Web of Conferences 8. 2113-2124.

Panckhurst R. (2007). Discours électronique médié : quelle évolution depuis une décennie ? In Gerbault J. (dir.). La langue du cyberespace : de la diversité aux normes. Paris : Harmattan. 121-136.

Paveau M.-A. (2015). Ce qui s'écrit dans les univers numériques. Itinéraires. Littérature, textes, cultures, 2014-1. 10.4000/itineraires. 2313

Petit M. (2010). Le discours spécialisé et le spécialisé du discours : repères pour l'analyse du discours en anglais de spécialité. E-rea. Revue électronique d'études sur le monde anglophone 8.1. https://doi.org/10.4000/erea.1400

Peytard J. (1993). D’une sémiotique de l'altération. Semen 8. 17-28.

Pélissier D. (2015). « Trace », vous avez vraiment dit « Trace ». En ligne (billet) : https:// presnumorg.hypotheses.org/76

Poudat C. (2006). Étude contrastive de l'article scientifique de revue linguistique dans une perspective d'analyse des genres. Thèse de doctorat. Université d'Orléans.

Quéré L. (2003). Le public comme forme et comme modalité d'expérience. In Cefaï D. \& Pasquier D. (dir.). Les Sens du public. Publics politiques, publics médiatiques. Paris : Presses Universitaires de France. 113-114.

Rastier F. (2011). La mesure et le grain. Sémantique de corpus. Paris : Honoré Champion.

Reboul S. (1995). Télématique et socioterminologie ». Meta : Journal des traducteurs 40(2). 296-307. En ligne : https://doi.org/10.7202/003918ar 
Reboul-Touré S. (2004). Écrire la vulgarisation scientifique aujourd'hui. Sciences, Médias et Société. 195-208.

Rey-Debove J. (1997). Le métalangage : étude linguistique du discours sur le langage. Paris : Colin (Collection U Série lettres).

Romeyer H. (2008). TIC et santé : entre information médicale et information de santé. tic\&société 2-1. En ligne : https://doi.org/10.4000/ticetsociete.365

Steuckardt A. (2015). Histoire de quelques correctifs formés sur dire. Langue française 186(2). 13-30.

Tutin A. (2007). Autour du lexique et de la phraséologie des écrits scientifiques. Revue française de linguistique appliquée XII(2). 5-14.

\section{NOTES}

1. Le terme " patient » désigne ici les personnes malades et, par extension, leurs proches et toute personne concernée par les informations délivrées par les guides.

2. Les analyses montrent que différents modèles sont nécessaires pour rendre compte de la réalité des discours de vulgarisation qui ne se laissent pas rassembler en une vison unitaire (Delavigne 2001).

3. http://www.nooj4nlp.net/pages/nooj.html

4. Un appel de la revue Corpus (2015) se faisait l'écho de l'abondance de ce type de structures lexico-syntaxiques plus ou moins figées.

5. Nous soulignons les marqueurs métalinguistiques.

6. La mise en forme et la graphie d'origine, ainsi que les usages spécifiques de la typographie et des ponctuèmes sont conservés tels quels.

7. En gras dans les exemples qui suivent.

8. http://www.tns-sofres.com/etudes/sante/190903_cancer.pdf

9. Pour une description du dispositif d'élaboration des brochures pour les patients et une description des acteurs, voir INCa (2013) et Delavigne (2017).

10. Sur un peu, voir Ducrot (2002).

\section{RÉSUMÉS}

Les terminologies peuvent être appréhendées de plusieurs manières. L'une d'entre elles est de les observer lorsqu'elles s'échappent de leur sphère d'usage habituelle. Afin de rétablir une intercompréhension, les discours destinés aux «non-experts » s'attachent à leur redonner du sens par un travail de négociation discursive qui s'emploie à faciliter l'émergence du sens des termes. L'analyse contrastive d'un corpus de documents médicaux, brochures pour les patients atteints de cancer et forums de discussion montre une économie spécifique des discours par des formes métalinguistiques à la fois semblables et spécifiques. Si le corpus révèle des usages attendus du métalinguistique comme outil explicatif, il semble bien qu'il ne s'y réduise pas. 
Terminologies can be examined in many ways. One of them is to observe when they escape their usual scope of usage. In order to reestablish intercomprehension, discourses that are intended for "non-experts" attempt to give them meaning through an effort of discursive negotiation that seeks to facilitate the emergence of the meaning of terms. The contrastive analysis of a corpus of medical documents, brochures for cancer patients and discussion forums shows a specific economy of discourse through metalinguistic forms that are both similar and specific. If the corpus reveals expected uses of metalinguistics as an explanatory tool, it appears that there is more to it.

\section{INDEX}

Mots-clés : socioterminologie, discours de vulgarisation, forums médicaux, activité métadiscursive, variation, ajustement sémantique, validation terminologique, expertise Keywords : socioterminology, popular discourses, medical forums, metadiscursive activity, variation, semantic adjustment, terminological validation, expertise

\section{AUTEUR}

\section{VALÉRIE DELAVIGNE}

Université Sorbonne nouvelle-Paris 3CLESTHIA EA 7345 